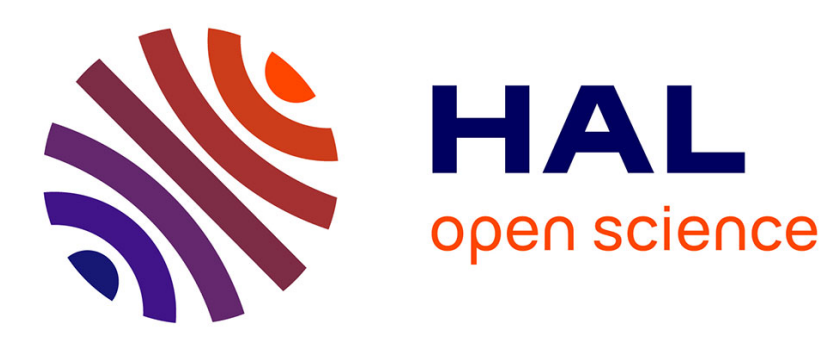

\title{
Scattering of flexural waves by a pit of quadratic profile inserted in an infinite thin plate
}

Omar Aklouche, Adrien Pelat, Sylvain Maugeais, François Gautier

\section{To cite this version:}

Omar Aklouche, Adrien Pelat, Sylvain Maugeais, François Gautier. Scattering of flexural waves by a pit of quadratic profile inserted in an infinite thin plate. Journal of Sound and Vibration, 2016, 375, pp.38-52. 10.1016/j.jsv.2016.04.034 . hal-02453174

\section{HAL Id: hal-02453174 \\ https://hal-univ-lemans.archives-ouvertes.fr/hal-02453174}

Submitted on 15 Dec 2020

HAL is a multi-disciplinary open access archive for the deposit and dissemination of scientific research documents, whether they are published or not. The documents may come from teaching and research institutions in France or abroad, or from public or private research centers.
L'archive ouverte pluridisciplinaire HAL, est destinée au dépôt et à la diffusion de documents scientifiques de niveau recherche, publiés ou non, émanant des établissements d'enseignement et de recherche français ou étrangers, des laboratoires publics ou privés. 


\title{
Scattering of flexural waves by a pit of quadratic profile inserted in an infinite thin plate
}

\author{
Omar Aklouche ${ }^{\mathrm{a}, \mathrm{b}, *}$, Adrien Pelat ${ }^{\mathrm{b}}$, Sylvain Maugeais ${ }^{\mathrm{c}}$, François Gautier ${ }^{\mathrm{b}}$ \\ ${ }^{a}$ Institut de Recherche Technologique Jules Verne, Chemin du Chaffault, 44340 \\ Bouguenais, France \\ ${ }^{b}$ Laboratoire d'Acoustique de l'Université du Maine, UMR CNRS 6613, Avenue Olivier \\ Messiaen, 72085 Le Mans, France \\ ${ }^{c}$ Laboratoire Manceau de Mathématiques, Université du Maine, Avenue Olivier \\ Messiaen, 72085 Le Mans, France
}

\begin{abstract}
An acoustic black hole $(\mathrm{ABH})$ is a pit of power law profile inserted in a plate with internal damping. Such a pit with varying thickness leads to local variations of wave propagation properties and has been found to be an efficient vibration damper. In this paper, $\mathrm{ABH}$ is seen as a penetrable obstacle and is studied through its scattering properties. A wave based model is developed within the framework of the Kirchhoff theory for a thin plate of locally varying thickness. It is shown that analytical solutions can be found in the case of a pit of quadratic profile and for uniform damping properties (without added layer). A major outcome of the model is the derivation of the dispersion relations giving a detailed analysis of the behavior of the waves within the ABH. Particularly, cut-on frequencies below which no wave propagates within the $\mathrm{ABH}$ are derived and thus give interpretation of the well known typical $\mathrm{ABH}$ efficiency threshold frequency for damping vibrations. The analysis is led from numerical computations of the dispersion curves, the scattering diagrams and the scattering cross-section, which are compared to the case of a simple hole. The results show that the $\mathrm{ABH}$ behaves as a resonant scatterer, which is a key outcome of this study. The so-called trapped modes, which describe free damped oscillations of the $\mathrm{ABH}$ are responsible for variations of the scattering cross-section with frequency. These variations are investigated thanks to a parametric study on the geometrical properties of the $\mathrm{ABH}$.
\end{abstract}

*Corresponding author 
Keywords: flexural vibration, wave scattering, acoustic black hole, trapped modes

\section{Introduction}

The lightening of mechanical structures constitutes an actual major issue in transport industry and in mechanical engineering since it generally leads to an increase in vibrational and acoustical levels. The usual passive methods, based on the use of viscoelastic coatings cannot always be used due to the additional mass they involve. In this context, a proposition consists in modifying the geometry of the structure to provide the so called acoustic black hole $(\mathrm{ABH})$ effect able to lead to large reduction of vibrational levels without mass increase of the structure. The ABH effect is related to bending wave properties in a beam of decreasing thickness and has been first theoretically shown by M. A. Mironov [1] in the context of geometrical acoustics. At the neighbourhood of the edge of a beam, if the thickness smoothly decreases, the wave slows down. If the tickness profile follows a power law and the thickness strictly vanishes at the edge, it can be shown that the needed travel time for a wave to reach the edge becomes infinite. Thus, the wave is not reflected at the edge so that the reflection coefficient vanishes. In practice, manufacturing $\mathrm{ABH}$ always involves non zero residual thickness at the edge (called truncation thickness) never small enough so that the reflection coefficient of the ABH area becomes large. V. Krylov showed [2] that the addition of a thin layer of viscoelastic material at the extremity can significantly reduce the effect of the truncation. This idea has been extended to two-dimensional structures at the edge of rectangular plates [3, 4] or inserted on plates of various geometries [5], which provides particularly attractive reductions of vibrational levels $[6,7]$.

When an incident wave interacts with an obstacle, a scattering phenomenon occurs : the obstacle behaves as secondary sources that radiate waves going out of itself. Over the last century, wave scattering by different kinds of obstacles has been extensively studied: P. Morse and H. Feshbach [8] gave the general theoretical framework for analyzing the scattering from cylinders and spheres of both electromagnetic and acoustic waves. An application to the case of flexural waves in thin plates has been done by C. Mow and Y. Pao [9] within the framework of the Kirchhoff theory to analyze scattering from a circular rigid inclusion. A. Norris and C. Vemula [10] studied analytically the scattering properties of soft and rigid obstacles inserted in an 
infinite thin plate. They gave results for the limiting cases of a circular rigid inclusion and a simple hole. The same authors [11] also analyzed the same scatterers within the framework of the Mindlin plate theory and pointed out the difference between these two plate theories. V. A. Squire and T. W. Dixon [12] applied a similar method to analyze the flexural wave scattering from a coated cylindrical obstacle in an infinite thin plate. In this case, since the scatterer is penetrable, the coupling between internal and external displacement fields is described by the continuity relations at the interface. The $\mathrm{ABH}$ belongs to this class of penetrable scatterers, for which there exists a field internal to the obstacle.

Although D. O'boy and V.V. Krylov [13] developed a numerical approach to the calculation of mobilities for a circular plate with a central $\mathrm{ABH}$, a theoretical description of the more general problem of the scattering of an incident plane wave from an $\mathrm{ABH}$ is not available in the litterature. This paper aims to apply the theoretical formulation from [10] to the particular case of a penetrable scatterer that consist in a circular pit of quadratic profile inserted in an infinite plate of uniform thickness. In order to reach a detailed understanding of the wave field inside and around the $\mathrm{ABH}$, an analytical solution of the problem is sought. Such an analytical solution requires the power-law profile to be quadratic and of uniform material properties, that is without added damping layer. Hence, the physical understanding provided by this work would then be usefull to develop other numerical approaches dedicated to the design of $\mathrm{ABH}$ inserted on industrial mechanical structures.

In section 2, the governing equations are presented and the resolution method is developed to model the scattering from an ABH. The known case of a simple hole is also recall to be compared with the ABH case. In section 3 , results from the model are presented to analyse the main $\mathrm{ABH}$ scattering characteristics. Trapped modes are obtained and are studied in more details in the section 4. Parametric studies of the scattering properties are reported in section 5. The conclusion finally gives the main outcomes and further works. 


\section{Model of flexural wave scattered by a circular acoustic black hole}

\subsection{Statement of the problem}

An infinite plate of constant thickness $h_{0}$, made in a material of mass density $\rho$, complex Young's modulus $E^{*}$ and Poisson's ratio $\nu$ is considered. A circular scatterer of external radius $b$ is placed at the origin of a polar coordinates system $(r, \theta)$. This scatterer can be a simple hole (Fig. 1-(a)) or an $\mathrm{ABH}$ of external radius $b$ and internal radius $a$ (Fig. 1-(b)).

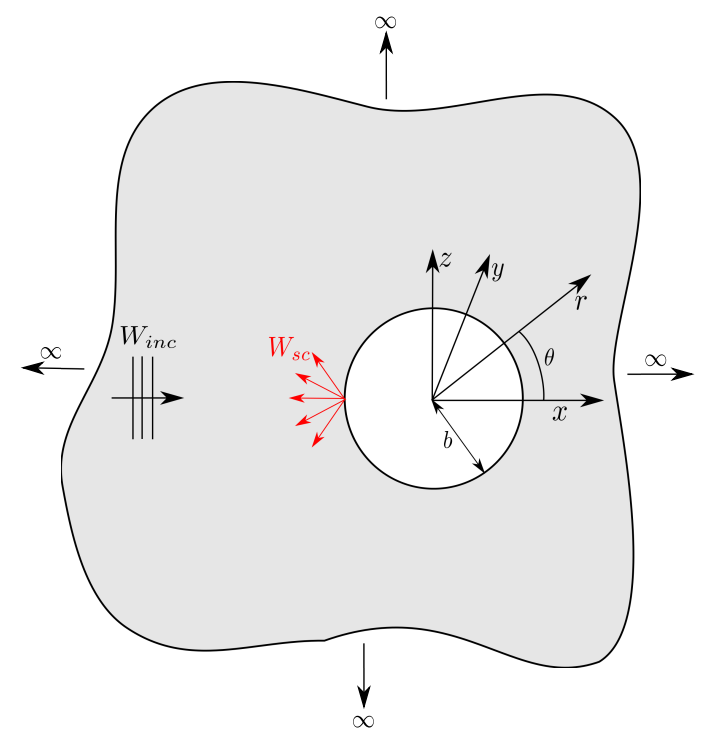

(a)

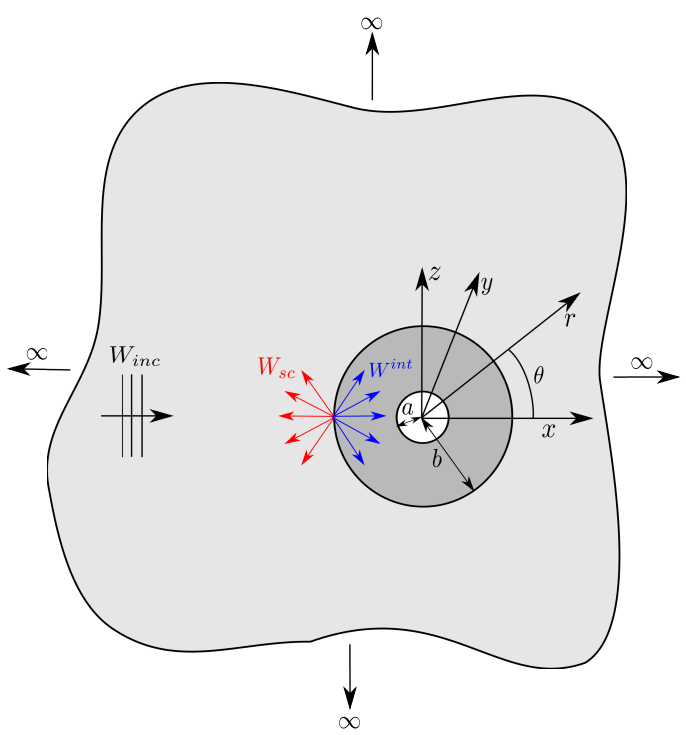

(b)

Figure 1: Scheme of the problem : scattering of an incident bending plane wave to (a) a simple hole or (b) an acoustic black hole inserted in an infinite thin plate.

The so called ABH scatterer consists in a pit of power low radial thickness profile given by

$$
h(r)=\left\{\begin{array}{ll}
h_{0}\left(\frac{r-a^{\prime}}{b-a^{\prime}}\right)^{m} \quad \text { with } m \geq 2, & a<r<b \\
h_{0}, & r=b
\end{array} .\right.
$$

Since ABH manufacturing process induces that zero thickness is impossible to reach at the edge in practice, two radii are defined. The so-called truncation radius $r=a$ represents the physical edge of the ABH central hole 
at which $h\left(a_{t}\right)=h_{t}>0$. The so-called zero thickness radius $r=a^{\prime}$ (with $\left.a^{\prime}<a\right)$ represents the fictitious edge of the central hole at which the thickness would vanish as shown in Fig. 2.

As represented in Fig. 1, an incident harmonic flexural plane wave $W_{\text {inc }}=$ $W_{0} e^{i k_{f} x} e^{-i \omega t}$, propagating along the $x$ direction is considered. The circular frequency is denoted $\omega, k_{f}$ is the natural flexural wavenumber in the plate of thickness $h_{0}$. Whatever the nature of the considered scatterer, $W_{\text {inc }}$ leads to a scattered field denoted $W_{s c}$.

In the following, an analytical model of the scattered field is proposed in order to evaluate scattering properties of an $\mathrm{ABH}$ and compare them with those of a simple hole scatterer.

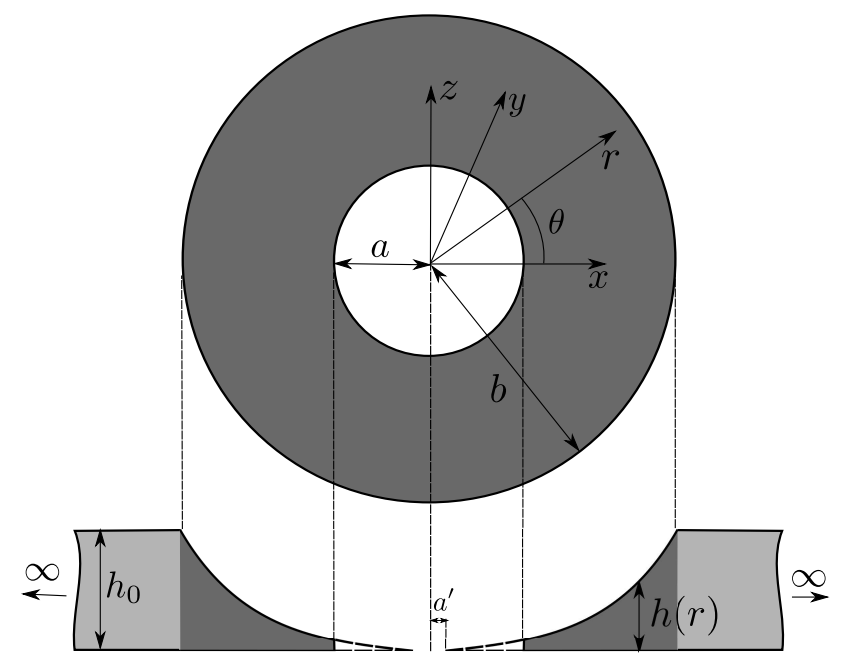

Figure 2: Acoustic Black Hole geometrical parameters considered in the model.

\subsection{Governing equations}

A Kirchhoff model for a harmonic motion is developed in the case of a thin plate of local varying thickness. This theoretical framework assumes that shear contribution is negligible which is valid if $\lambda>6 h$ at each point of the plate [14]. If this low frequency assumption is not satisfied, a Midlin model should be used [15]. Moreover, the out of plane displacement is supposed to be small compared with the thickness leading to the linear Kirchhoff model. If this assumption $w / h>>1$ is not satisfied, a Von Karman model 
should be used [16]. The ABH geometries and frequency ranges chosen in this paper are such that the Kirchhoff theory is valid.

Outside the scatterer $(r>b)$, the flexural displacement field $W^{e x t}(r, \theta)$, called external field, satisfies to the equation:

$$
\left(\Delta^{2}-k_{f}^{4}\right) W^{e x t}=0, \quad \forall r \geq b,
$$

where $\Delta$ is the Laplacian operator in cylindrical coordinates. The wave number $k_{f}=\sqrt[4]{\frac{\rho h_{0}}{D_{0}}} \sqrt{\omega}$ is the complex flexural wave number outside the scatterer $\left(\Re\left[k_{f}\right]>0\right.$ and $\left.\Im\left[k_{f}\right]>0\right)$. The material density is denoted by $\rho$ and the bending stiffness is $D_{0}=\frac{E^{*} h_{0}^{3}}{12\left(1-\nu^{2}\right)}$, where the complex Young's modulus is $E^{*}=E_{0}(1-i \eta), E_{0}$ and $\eta$ being respectively the elastic Young's modulus and the damping loss factor of the plate material.

Inside the ABH scatterer $(a<r<b)$, the thickness decreases according to Eq. (1). The internal bending stiffness is independent from $\theta$ and only varies with respect to $r$ as $D^{i n t}(r)=D_{0}\left(\frac{r-a^{\prime}}{b-a^{\prime}}\right)^{3 m}$. Hence, the flexural displacement field $W^{\text {int }}(r, \theta)$, called internal field, satisfies to the equation of motion $[17,13]$

$$
\nabla^{2}\left[D^{i n t} \nabla^{2} W^{i n t}\right]-(1-\nu) \diamond^{4}\left\{D^{i n t}, W^{i n t}\right\}-\rho h \omega^{2} W^{i n t}=0, \quad a<r<b,
$$

where the $\diamond$ operator is defined by

$$
\diamond^{4}\left\{D^{i n t}, W^{i n t}\right\}=\frac{\partial^{2} D^{i n t}}{\partial r^{2}}\left[\frac{1}{r} \frac{\partial W^{i n t}}{\partial r}+\frac{1}{r^{2}} \frac{\partial^{2} W^{i n t}}{\partial \theta^{2}}\right]+\frac{\partial^{2} W^{i n t}}{\partial r^{2}}\left[\frac{1}{r} \frac{\partial D^{i n t}}{\partial r}\right]
$$

\subsection{Boundary and continuity conditions}

At the interface $(r=b)$ between the ABH scatterer and the homogeneous plate, the displacement field $W$, the slope $\Theta$, the bending moment $M$ and 
the shear force $V$ are continuous, which respectively leads to

$$
\begin{aligned}
& \left.W^{e x t}\right|_{r=b}=\left.W^{i n t}\right|_{r=b}, \\
& \left.\Theta\left(W^{e x t}\right)\right|_{r=b}=\left.\Theta\left(W^{i n t}\right)\right|_{r=b}, \\
& \left.M\left(W^{\text {ext }}\right)\right|_{r=b}=\left.M\left(W^{i n t}\right)\right|_{r=b}, \\
& \left.V\left(W^{e x t}\right)\right|_{r=b}=\left.V\left(W^{\text {int }}\right)\right|_{r=b} .
\end{aligned}
$$

The $\Theta, M$ and $V$ operators are given by

$$
\begin{gathered}
\Theta(\cdot)=\frac{\partial(\cdot)}{\partial r} \\
M(\cdot)=-D_{0}\left\{\frac{\partial^{2}(\cdot)}{\partial r^{2}}+\nu\left[\frac{1}{r} \frac{\partial(\cdot)}{\partial r}+\frac{1}{r^{2}} \frac{\partial^{2}(\cdot)}{\partial \theta^{2}}\right]\right\} \\
V(\cdot)=-\left(D^{i n t}\right) \frac{\partial^{3}(\cdot)}{\partial r^{3}}-\left(\frac{\partial D^{i n t}}{\partial r}+\frac{D^{i n t}}{r}\right) \frac{\partial^{2}(\cdot)}{\partial r^{2}}+\left(\frac{D^{i n t}}{r^{2}}-\frac{\nu}{r} \frac{\partial D^{i n t}}{\partial r}\right) \frac{\partial(\cdot)}{\partial r} \\
-\left(\frac{D^{i n t}(2-\nu)}{r^{2}}\right) \frac{\partial^{3}(\cdot)}{\partial r \partial \theta^{2}}+\left(\frac{D^{i n t}(3-\nu)}{r^{3}}-\frac{\partial D^{i n t}}{r^{2}}\right) \frac{\partial^{2}(\cdot)}{\partial \theta^{2}}
\end{gathered}
$$

Note that when the rigidity of the plate is constant $\left(D^{i n t}=D_{0}\right)$, Eq. (8) is reduced to

$$
V_{0}(\cdot)=-D 0\left\{\frac{\partial}{\partial r} \nabla^{2}(\cdot)+(1-\nu) \frac{1}{r^{2}} \frac{\partial^{2}}{\partial \theta^{2}}\left[\frac{\partial(\cdot)}{\partial r}-\frac{(\cdot)}{r}\right]\right\}, \quad \forall r \geq b
$$

At the edge of the ABH central hole $(r=a)$, the free boundary condition gives

$$
\begin{aligned}
& \left.M\left(W^{i n t}\right)\right|_{r=a}=0 \\
& \left.V\left(W^{i n t}\right)\right|_{r=a}=0
\end{aligned}
$$

Finally, for the $\mathrm{ABH}$ case, the problem to solve is defined by the equation of motion (3) associated to the continuity conditions (5), the boundary conditions (10) and the excitation condition $W_{i n c}=W_{0} e^{i k_{f} x}$. 
For the case of a simple hole, no internal field has to be taken into account since the scatterer is not penetrable. The corresponding free boundary conditions at $r=b$ are written as

$$
\begin{aligned}
& \left.M\left(W^{e x t}\right)\right|_{r=b}=0, \\
& \left.V_{0}\left(W^{e x t}\right)\right|_{r=b}=0,
\end{aligned}
$$

and the problem to solve is defined by the equation of motion (2) associated to boundary conditions (11) and excitation condition $W_{i n c}=W_{0} e^{i k_{f} x}$.

\subsection{Resolution method}

\subsubsection{External field $(r>b)$}

Outside the $\mathrm{ABH}(r>b)$, the total displacement field $W^{e x t}(\widehat{r}, \theta)$ is a superposition of the incident plane wave $W_{\text {inc }}(\widehat{r}, \theta)$ and the scattered field $W_{s c}(\widehat{r}, \theta)$ :

$$
W^{e x t}(\widehat{r}, \theta)=W_{i n c}(\widehat{r}, \theta)+W_{s c}(\widehat{r}, \theta), \text { with } \widehat{r}=r-a^{\prime},
$$

where the incident plane wave can be expanded using Bessel functions of the first kind,

$$
W_{i n c}=W_{0} \sum_{n=0}^{\infty} \varepsilon_{n} i^{n} J_{n}\left(k_{f} \widehat{r}\right) \cos (n \theta), \quad \varepsilon_{n}=\left\{\begin{array}{ll}
1, & n=0 \\
2, & n>0
\end{array} .\right.
$$

The scattered field takes the form $[9,10]$

$$
W_{s c}=\sum_{n=0}^{\infty}\left[A_{n} H_{n}^{(1)}\left(k_{f} \widehat{r}\right)+B_{n} K_{n}\left(k_{f} \widehat{r}\right)\right] \cos (n \theta), \quad \forall r>b,
$$

which is the general solution of Eq. (2) that takes into account only outgoing propagating and evanescent waves, represented by Hankel function of the first kind $H_{n}^{(1)}$ and modified Bessel function of the second kind $K_{n}$ of the order $n$ respectively $[18,19]$.

Finally, the total external field is written

$$
W^{e x t}=W_{0} \sum_{n=0}^{\infty} \varepsilon_{n} i^{n} J_{n}\left(k_{f} \widehat{r}\right) \cos (n \theta)+\sum_{n=0}^{\infty}\left[A_{n} H_{n}^{(1)}\left(k_{f} \widehat{r}\right)+B_{n} K_{n}\left(k_{f} \widehat{r}\right)\right] \cos (n \theta), \quad \forall r>b
$$


The constants $A_{n}$ and $B_{n}$ for each circumferential order $n$ are found for the simple hole case by substituting Eq. (15) into the free boundary conditions (11). The obtained system of two equations is then analytically solved [10] (see details in Appendix A). For the ABH case, the internal field has to be described, which is done in the next paragraph.

\subsubsection{Displacement field inside the $A B H(a<r<b)$}

Inside the ABH, the internal field satisfies to the Eq. (3). Such solution is $2 \pi$ periodic and can be written as a Fourier series expansion

$$
W^{i n t}(\widehat{r}, \theta)=\sum_{n=0}^{\infty} W_{n}^{i n t}(\widehat{r}) \cos (n \theta)
$$

where the $x$ axis is the reference axis for the angle $\theta$ and $\widehat{r}$ is defined in Eq. (12).

Substituting (16) into (3) and applying the orthogonality property of the functions $\cos (n \theta)$ lead to the following equation satisfied by $W_{n}^{i n t}(\widehat{r})$ for each circumferential order $n$ :

$$
\begin{aligned}
& \frac{\partial^{4} W_{n}^{i n t}}{\partial \widehat{r}^{4}}+\left[\frac{2}{D^{i n t}} \frac{\partial D^{i n t}}{\partial \widehat{r}}+\frac{2}{\widehat{r}}\right] \frac{\partial^{3} W_{n}^{i n t}}{\partial \widehat{r}^{3}}+\left[\frac{1}{D^{i n t}} \frac{\partial^{2} D^{i n t}}{\partial \widehat{r}^{2}}+\frac{2+\nu}{\widehat{r} D^{i n t}} \frac{\partial D^{i n t}}{\partial \widehat{r}}-\frac{2 n^{2}+1}{\widehat{r}^{2}}\right] \frac{\partial^{2} W_{n}^{i n t}}{\partial \widehat{r}^{2}} \\
& +\left[\frac{\nu}{\widehat{r} D^{i n t}} \frac{\partial^{2} D^{i n t}}{\partial \widehat{r}^{2}}-\frac{2 n^{2}+1}{\widehat{r}^{2} D^{i n t}} \frac{\partial D^{i n t}}{\partial \widehat{r}}+\frac{2 n^{2}+1}{\widehat{r}^{3}}\right] \frac{\partial W_{n}^{i n t}}{\partial \widehat{r}} \\
& -\left[\frac{\nu n^{2}}{\widehat{r}^{2} D^{i n t}} \frac{\partial^{2} D^{i n t}}{\partial \widehat{r}^{2}}-\frac{3 n^{2}}{\widehat{r}^{3} D^{i n t}} \frac{\partial D^{i n t}}{\partial \widehat{r}}+\frac{n^{2}\left(4-n^{2}\right)}{\widehat{r}^{4}}+\frac{\rho h(\widehat{r}) \omega^{2}}{D^{i n t}}\right] W_{n}^{i n t}=0 .
\end{aligned}
$$

Defining $D^{i n t}(\widehat{r})=D_{0}\left(\frac{r-a^{\prime}}{b-a^{\prime}}\right)^{3 m}[13,20]$, Eq. (17) becomes 


$$
\begin{aligned}
& \widehat{r}^{4} \frac{\partial^{4} W_{n}^{i n t}}{\partial \widehat{r}^{4}}+\widehat{r}^{3}[2+6 m] \frac{\partial^{3} W_{n}^{i n t}}{\partial \widehat{r}^{3}}+\widehat{r}^{2}\left[3 m(3 m+1+\nu)-\left(1+2 n^{2}\right)\right] \frac{\partial^{2} W_{n}^{\text {int }}}{\partial \widehat{r}^{2}} \\
& +\widehat{r}\left[3 m(3 m-1) \nu-3 m\left(1+2 n^{2}\right)+\left(1+2 n^{2}\right)\right] \frac{\partial W_{n}^{\text {int }}}{\partial \widehat{r}} \\
& +\left[3 m\left(3 n^{2}-(3 m-1) \nu n^{2}\right)+n^{2}\left(n^{2}-4\right)-\frac{12 \rho \omega^{2}\left(1-\nu^{2}\right)}{E^{*} h_{0}^{2}\left(b-a^{\prime}\right)^{-2 m}} \widehat{r}^{4-2 m}\right] W_{n}^{\text {int }}=0 .
\end{aligned}
$$

\subsubsection{Quadratic thickness profile $(m=2)$}

In the particular case of a quadratic profile $(m=2)$, the last term of Eq. (18) does not depend on $\widehat{r}$ anymore. This differential equation can be solved using the change of space variable

$$
\widehat{r}=r_{0} e^{\widehat{z}}
$$

implying $\frac{\partial(\cdot)}{\partial \widehat{r}}=\frac{1}{\widehat{r}} \frac{\partial(\cdot)}{\partial \widehat{z}}$, where $\widehat{z}=\frac{z}{r_{0}}$ is a dimensionless parameter and $r_{0}=b-a^{\prime}$. Eq. (18) is thus written as a differential equation with constant coefficients:

$\alpha_{4} \frac{\partial^{4} W_{n}^{i n t}(\widehat{z})}{\partial \widehat{z}^{4}}+\alpha_{3} \frac{\partial^{3} W_{n}^{i n t}(\widehat{z})}{\partial \widehat{z}^{3}}+\alpha_{2} \frac{\partial^{2} W_{n}^{i n t}(\widehat{z})}{\partial \widehat{z}^{2}}+\alpha_{1} \frac{\partial W_{n}^{i n t}(\widehat{z})}{\partial \widehat{z}}+\alpha_{0} W_{n}^{\text {int }}(\widehat{z})=0$,

in which $\alpha_{4}=1, \alpha_{3}=8, \alpha_{2}=10+6 \nu-2 n^{2}, \alpha_{1}=-24+24 \nu-8 n^{2}$, $\alpha_{0}=n^{2}\left(n^{2}-30 \nu+14\right)-\frac{12 \rho \omega^{2}\left(1-\nu^{2}\right)}{E^{*} h_{0}^{2} r_{0}^{-4}}$. Looking for solutions $W_{n}^{\text {int }}(\widehat{z})$ proportional to $e^{K \widehat{z}}$ ( $K$ being dimensionless), the following characteristic equation is derived :

$$
\alpha_{4} K^{4}+\alpha_{3} K^{3}+\alpha_{2} K^{2}+\alpha_{1} K+\alpha_{0}=0 .
$$

For each circumferential order $n$, Eq. (21) has four roots $K_{p, n}(p=$ $1,2,3,4)$ that can be analytically found from the Ludovico Ferrari's method 
[21] :

$$
K_{p, n}=-2 \mp \sqrt{7-3 \nu+n^{2} \pm \sqrt{8 n^{2}(3 \nu-1)+9(1-\nu)^{2}+\frac{12 \rho \omega^{2}\left(1-\nu^{2}\right)}{E^{*} h_{0}^{2} r_{0}^{-4}}}}
$$

where $K_{1, n}, K_{2, n}, K_{3, n}$ and $K_{4, n}$ are obtained from (22), taking the sign combinations $(-,+),(+,+),(-,-)$ and $(+,-)$, respectively.

The expression of the displacement field inside the $\mathrm{ABH}$ is finally given by

$$
W^{i n t}=\sum_{n=0}^{\infty} \sum_{p=1}^{4}\left[C_{p, n} e^{i k_{p, n} z}\right] \cos (n \theta), \quad k_{p, n}=\frac{K_{p, n}}{i r_{0}} .
$$

The coefficients $C_{p, n}, A_{n}$ and $B_{n}$ for each circumferential order $n$ are found for the ABH by substituing Eq. (23) into free boundary conditions (10) and Eq. (15) into continuity equations (5). The obtained system of six equations (see details in Appendix B) is then numerically solved.

\section{Numerical results}

All the numerical results presented in section 3 are obtained for an aluminum plate which geometrical and material properties are given in table 1 . These geometrical settings involve a thickness ratio $h_{t} / h_{0}=1 / 100$ with $h_{t}$ the truncation thickness of the ABH..

\begin{tabular}{|c|l|c|ll|}
\hline Material properties & Values & Geometric properties & Values & \\
\hline$\rho$ & $2700\left(\mathrm{~kg} / \mathrm{m}^{3}\right)$ & $a^{\prime}$ & $10^{-3}$ & $(\mathrm{~m})$ \\
$\nu$ & 0.33 & $a$ & $5.10^{-3}$ & $(\mathrm{~m})$ \\
$E_{0}$ & $69(\mathrm{GPa})$ & $b$ & $6.10^{-2}$ & $(\mathrm{~m})$ \\
$\eta$ & $10^{-3}$ & $h_{0}$ & $1,5.10^{-3}$ & $(\mathrm{~m})$ \\
& & $m$ & 2 & \\
\hline
\end{tabular}

Table 1: Parameters of the numerical computation of the simulated ABH. 


\subsection{Dispersion relations}

The model presented in section 2 shows that in the transformed space $\widehat{z}$ defined from the change of space variable (19), the ABH can be seen as a propagation medium in which four waves can be derived. The dispersion of these four waves is studied in Fig. 3 in which real and imaginary parts of the dimensionless wave numbers $k_{p, n} b(p=1$ to 4$)$ are plotted as a function of dimensionless flexural wave number $k_{f} b$ ( $b$ being the external radius of the $\mathrm{ABH})$. For readability, only the fundamental circumferential order $(n=0)$ is represented in Fig. 3.
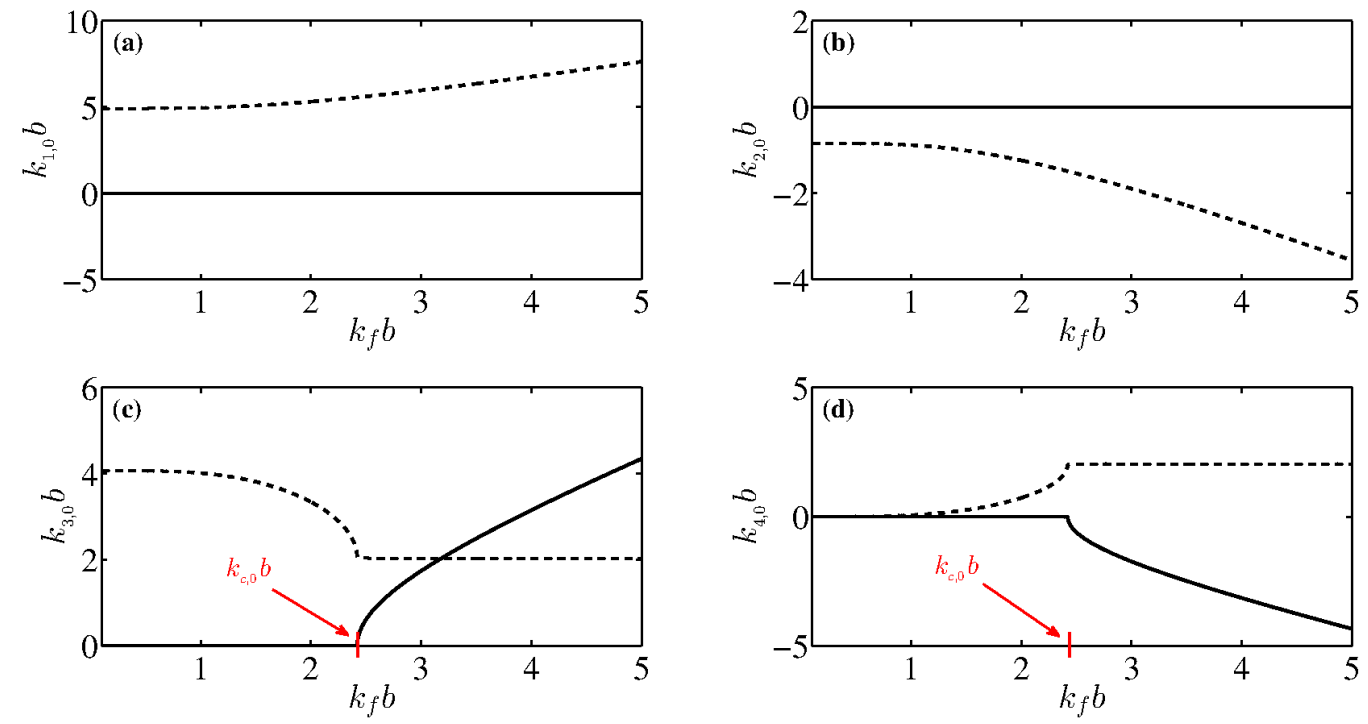

Figure 3: Dispersion curves of the ABH. Real (- - and imaginary $(---)$ parts of $k_{1,0}$, $k_{2,0}, k_{3,0}$ and $k_{4,0}$ are plotted versus the dimensionless wave number $k_{f} b$ defined in the uniform area.

From time convention $e^{-i w t}$, a wave associated to a wave number $k_{p, n}$ for which $\Re\left\{k_{p, n}\right\}>0$ corresponds to a so called divergent (or outgoing) wave, i.e a wave travelling in the increasing $x$ direction. Then, $\Re\left\{k_{p, n}\right\}<0$ corresponds to a convergent (or ingoing) wave. The imaginary part $\Im\left\{k_{p, n}\right\}$ results in an increasing $\left(\Im\left\{k_{p, n}\right\}<0\right)$ or decreasing $\left(\Im\left\{k_{p, n}\right\}>0\right)$ exponential envelope in the increasing $x$ direction.

The Figs. 3(a) and 3(b) show that $k_{1,0}$ and $k_{2,0}$ correspond to convergent and divergent evanescent waves, respectively. The plots of $k_{3,0}$ (Fig. 3-(c)) and 
$k_{4,0}$ (Fig. 3-(d)) display a dimensionless cut-on wavenumber for the order $n$ denoted $k_{c, n} b$ whose expression is derived from Eq. (22) :

$$
k_{c, n} b=\frac{b}{b-a^{\prime}} \sqrt[4]{n^{2}\left(n^{2}-30 \nu+22\right)-24 \nu+40} .
$$

The corresponding cut-on frequency $f_{c, n}$ can be derived from Eq. (24) as

$$
f_{c, n}=\frac{h_{0}}{2 \pi\left(b-a^{\prime}\right)^{2}} \sqrt{\frac{E_{0}\left[n^{2}\left(n^{2}-30 \nu+22\right)-24 \nu+40\right]}{12 \rho\left(1-\nu^{2}\right)}} .
$$

Below $k_{c, 0}$, the wavenumber $k_{3,0}$ corresponds to a divergent evanescent wave that becomes propagative and still exponentially attenuated above $k_{c, 0}$. The case of $k_{4,0}$ is particularly interesting and illustrative of the $\mathrm{ABH}$ effect : at low frequencies (for wavenumbers below $k_{c, 0}$ ), the wave corresponding to $k_{4,0}$ is a convergent evanescent wave that becomes propagative and exponentially amplified above $k_{c, 0} b \approx 2.42$ for the simulated ABH. This analysis gives interpretations of experimental and numerical results from the literature $[6,7]$, where it is shown that the $\mathrm{ABH}$ becomes efficient from a threshold frequency, also called starting frequency in [22], which corresponds here to the first cut-on frequency $f_{c, 0}$. Above this cut-on frequency, propagating waves inside the ABH appear, leading to local internal resonances.

Fig. 4 shows that analysis from Fig. 3 can be generalized for all circumferential orders $n$ since a cut-on wave number $k_{c, n}$ always exists following Eq. (24). The wavenumber $k_{c, n}$ discriminates similar evanescent (below $k_{c, n}$ ) or propagative (above $k_{c, n}$ ) behaviours shown in Fig. 3. Otherwise, for each circumferential order $n$, two evanescent waves (not represented in Fig. 4) are found whatever the frequency.

\subsection{Displacement field}

The total displacement field can be computed by solving the linear system in Eq. (B.5). Fig. 5(a) displays the modulus (dB scale) of the displacement field at four frequencies $f \approx 26 \mathrm{~Hz}, 410 \mathrm{~Hz}, 923 \mathrm{~Hz}$ and $2563 \mathrm{~Hz}$, corresponding to the dimensionless wavenumbers $k_{f} b=0.5,2,3$ and 5 respectively. As expected and already shown in previous works $[6,7]$, the vibrational field inside the $\mathrm{ABH}$ is nearly uniform at low frequencies: since no wave is propagative below the first cut-on frequency, the pit is almost inactive such that no wave pattern appears (see $\left.k_{f} b=0.5\right)$. At higher frequencies $\left(k_{f} b=3,5\right)$, the typical drop of the wavelength and the grow of the magnitude is observed 

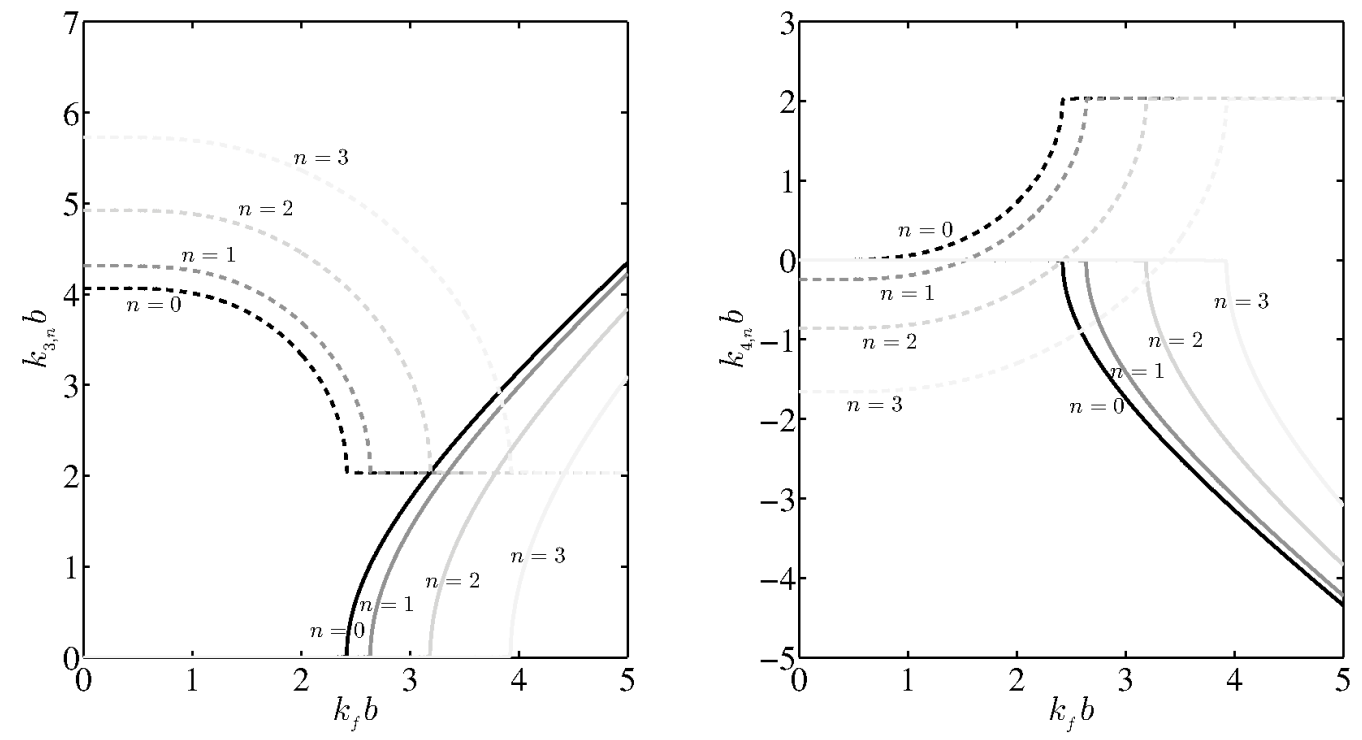

Figure 4: Dispersion curves of $k_{3, n}$ for the circumferential orders $n=0,1,2,3$. Real ( - ) and imaginary $(---)$ parts of $k_{3, n}$ are plotted versus the dimensionless wave number $k_{f} b$ defined in the uniform area.

as expected. Moreover, the field shows more complex interference patterns with increasing frequency due to the increasing number of propagative waves associated to circumferential orders $n$.

The so called scattering pattern function $f(\theta)$ that corresponds to the far field magnitude of the scattered field $W^{s c}(r>>1)$ is also shown in Fig. 5(b) as polar plots. $f(\theta)$ is the asymptotic expression of Eq. (14) for which $r \rightarrow \infty$ and in which evanescent waves are thus neglected [10]:

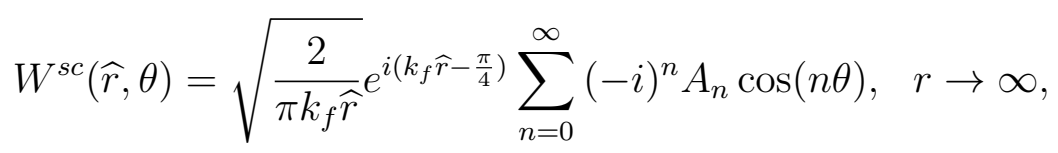

and can be rewritten as

$$
W^{s c}(\widehat{r}, \theta)=\frac{1}{\sqrt{2 \widehat{r}}} e^{i\left(k_{f} \widehat{r}-\pi / 4\right)} f(\theta), \quad r \rightarrow \infty,
$$



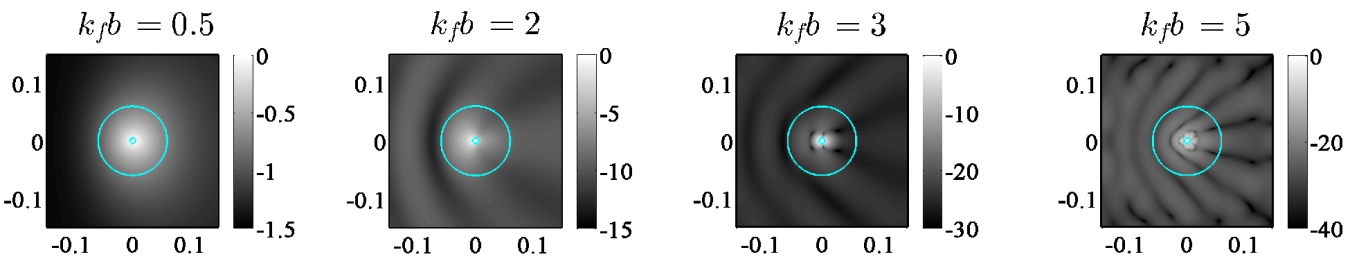

(a)
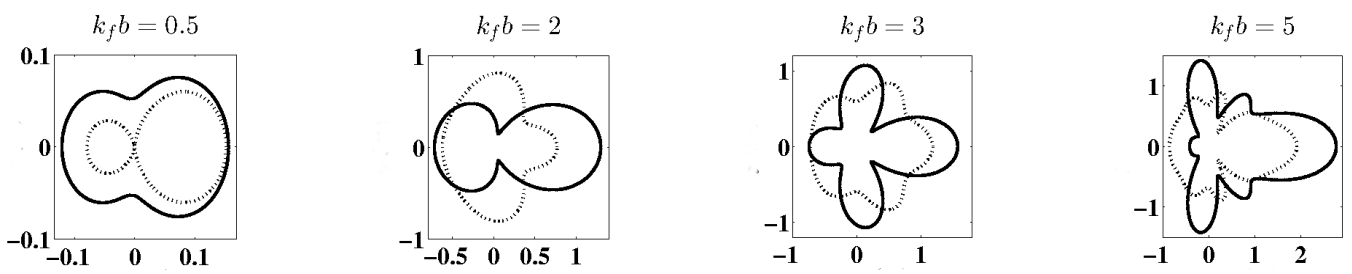

(b)

Figure 5: (a) Modulus (dB scale) of the displacement field of an infinite thin plate with an $\mathrm{ABH}$ at frequencies of the incident wave corresponding to $k_{f} b=0.5, k_{f} b=2, k_{f} b=3$ and $k_{f} b=5$. The blue circles indicate internal and external $\mathrm{ABH}$ boundaries. For each frequency, the displacement field is normalized to its maximal value. (b) Associated scattering pattern functions (defined in Eq. (28)) of the ABH (- ) and of a simple hole (.........) of thevsame external radius.

with

$$
f(\theta)=\frac{2}{\sqrt{\pi k_{f}}} \sum_{n=0}^{\infty}(-i)^{n} A_{n} \cos (n \theta) .
$$

Fig. 5(b) shows that at low frequencies $\left(k_{f} b=0.5\right)$, the $\mathrm{ABH}$, being inactive, induces a low scattering effect (the magnitude of the scattered field is less than $10 \%$ of the incident wave) and exhibits roughly the same behavior as the simple hole. At higher frequencies $\left(k_{f} b=2,3,5\right)$, the scattered field is in the range of magnitude than the incident wave and exhibits lobs of directivity as complex as the wave field is carried by an increasing number of wave associated to circumferential orders.

\subsection{Scattering cross-section}

The scattering-cross section $Q_{s c}$ is classically used to evaluate the amount of energy scattered by an obstacle. This quantity is defined as the ratio between the total energy flux of the scattered field to the one per unit length 
of the incident field [10]. In the context of this article, it can be shown that $Q_{s c}$ is homogeneous to a length and is given by

$$
Q_{s c}=\frac{1}{2} \int_{0}^{2 \pi}|f(\theta)|^{2} d \theta .
$$

$Q_{s c}$ of the simulated $\mathrm{ABH}$ and simple hole of the same external radius $b$ are plotted in Fig. 6. At a low frequency (zoomed view of Fig. 6(b)), both the $\mathrm{ABH}$ and the simple nearly behave the same : the wavelength being large compared with the external radius $b$, both obstacles do almost not scatter.

Above the cut-on dimensionless wave number $k_{c, 0} b=2.42$, the two obstacles behave very differently. While $Q_{s c}$ of the simple hole smoothly increases with increased frequency [10], the ABH shows strong variations. Particularly, the local maxima show that the $\mathrm{ABH}$ induce at given frequencies rather much scattering than the simple hole. The variations of $Q_{s c}$ is the result of a complex combination of the elementary scattering cross-sections associated to each circumferential order. The plots of Fig. 7 for the orders $n=0,1,2$ show that the maxima of the elementary scattering cross-sections are associated to resonances of trapped modes of the $\mathrm{ABH}$. Indeed, above each cut-on frequency $f_{c, n}$, wave interferences lead to series of trapped modes associated to the order $n$. Trapped modes are studied in section 4 .

At high frequencies $\left(k_{f} b>5\right), Q_{s c}$ of the $\mathrm{ABH}$ varies around the simple hole which tends to an asymptotic value (corresponding to approximately the half perimeter $\pi b$ ). These variations show a certain periodicity versus frequency which can be interpreted from the asymptotic expression of (24) : when $n \gg 1$ (at large frequencies), $k_{c, n} b \approx \frac{b}{b-a^{\prime}} n$ such that the cut-on frequencies become harmonic one each other which leads to nearly repeated patterns of resonances.

\section{Trapped modes of flexural waves}

The presence of a penetrable scatterer in an infinite medium can give rise to local resonance phenomena, related to its so called trapped modes. These modes correspond to the free oscillations of finite energy localized around 


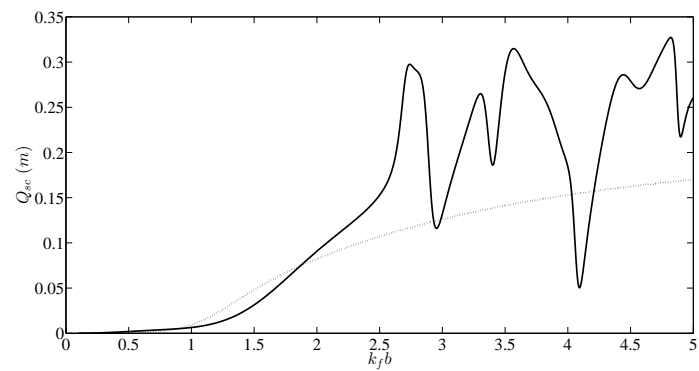

(a)

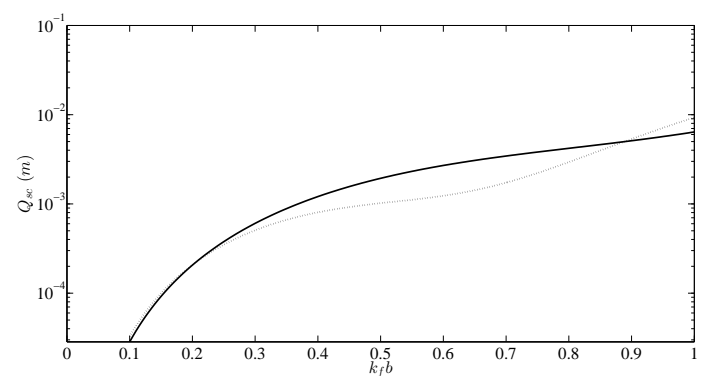

(b)

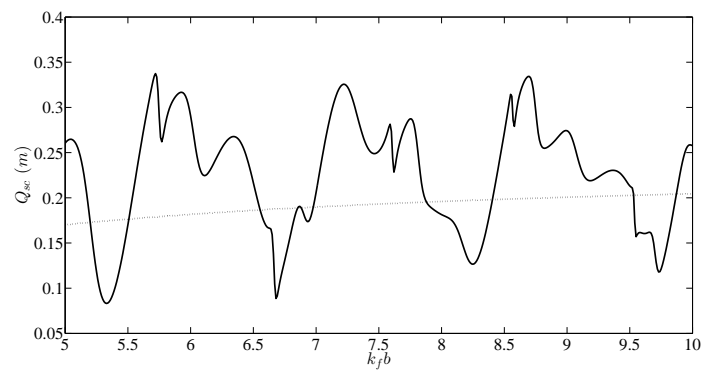

(c)

Figure 6: (a) Scattering cross-sections of an ABH (- ${ }^{-}$) and a simple hole (........). (b) Zoomed view of Fig. 6(a) in the low frequency range (logarithmic scale). (c) Scattering cross-sections of the $\mathrm{ABH}$ and simple hole for dimensionless wavenumbers $k_{f} b$ between 5 and 10, computed for loss factor $\eta=0.001$.

and eventually inside the scatterer, if penetrable. trapped modes have been observed and studied in different fields of physics. In the case of a twodimensional acoustic waveguide, M. Callan et al. [23] studied the local free oscillations in the vicinity of a circular rigid obstacle. P. J. Cobelli et al. [24] 


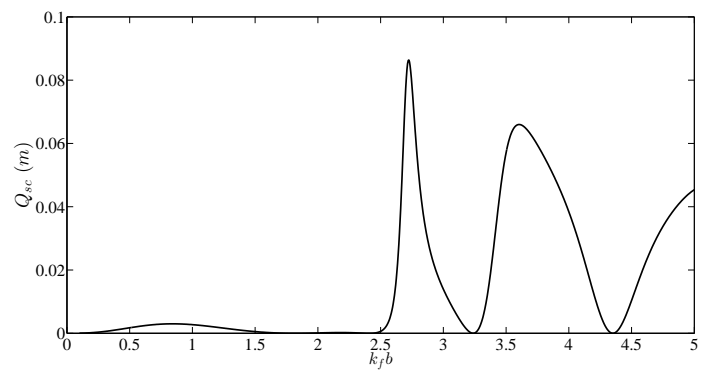

(a)

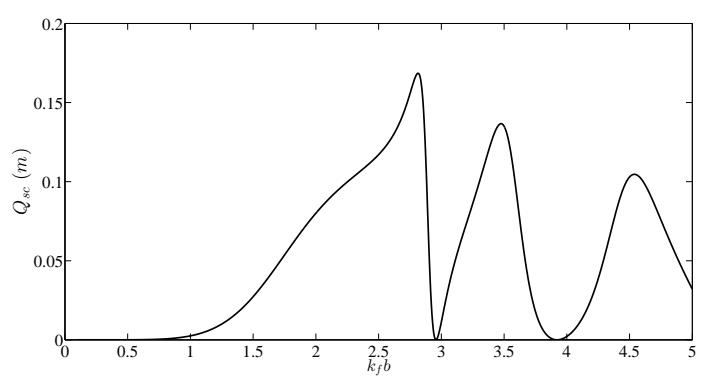

(b)

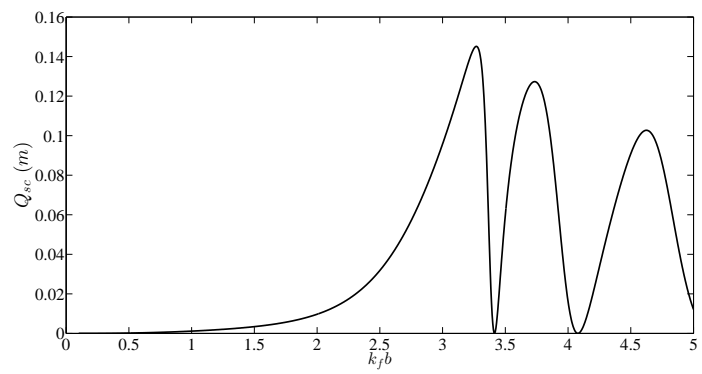

(c)

Figure 7: Elementary scattering cross-section of the simulated $\mathrm{ABH}$ for the three first circumferential orders (a) $n=0$, (b) $n=1$ and (c) $n=2$.

experimentally observed trapped modes in a water channel in which a rigid circular cylinder is inserted. R. Porter [25] shown that trapped modes are found numerically when considering a simple hole with free edge, placed in a mechanical waveguide made with a thin plate of infinite length with parallel simply supported edges. O. Xeridat et al. [26] analyzed the Lamb waves 
scattering and local resonances of a non through hole in a plate.

\subsection{Homogeneous problem}

By definition, the trapped modes are the solutions of a homogeneous problem, given in the case of an $\mathrm{ABH}$ scatterer by the matrix equation

$$
M . X=0,
$$

where $\boldsymbol{M}$ is defined from the continuity and boundary conditions, as detailed in the appendix B. Eigenvalues of Eq. (30) are found in the complex

$k b$ plane by numerically locating the zeros of $\operatorname{Det}(\boldsymbol{M})$. Then, mode shapes are obtained from the eigenvectors $\boldsymbol{X}$ associated to found eigenvalues.

\subsection{Typical results}

The Fig. 8 shows examples of trapped modes patterns. The relation between these modes and the scattering cross-section of the $\mathrm{ABH}$ is here highlighted: maximal values of $Q s c$ in Fig. 6-(a) correspond to real parts of the eigenvalues $k b$ in Fig. 8. For example, maxima of $Q s c$ at $k_{f} b \approx 2.73$ and $k_{f} b \approx 3.56$ are associated to the modes for which $k b=2.71-0.07 i$ and $k b=3.56-0.2 i$ respectively.

\section{Parametric studies}

The material and geometrical parameters of the $\mathrm{ABH}$ are numerous and their effect on the scatering properties would be evaluated in order to set some designing rules able to taylor the $\mathrm{ABH}$ scattering properties to specific mechanical engineering applications. Since the response of the ABH is the result of a complex combination of circumferential orders, to find explicit links between the $\mathrm{ABH}$ design and its scattering propoerties is difficult in the framework of this model. Nevertheless, parametric studies can be easily handled to give trends. This section focuses on the influence of the material loss factor $\eta$, the truncation thickness $h_{t}$ and the truncation radius $a^{\prime}$ of the ABH. From Eq. (1) with $m=2$, the following relation between $h_{t}$ and $a^{\prime}$ can be derived to drive the geometrical parameter settings of sections 5.2 and 5.3:

$$
a=a^{\prime}\left(1-\sqrt{\frac{h_{t}}{h_{0}}}\right)+b\left(\sqrt{\frac{h_{t}}{h_{0}}}\right)
$$



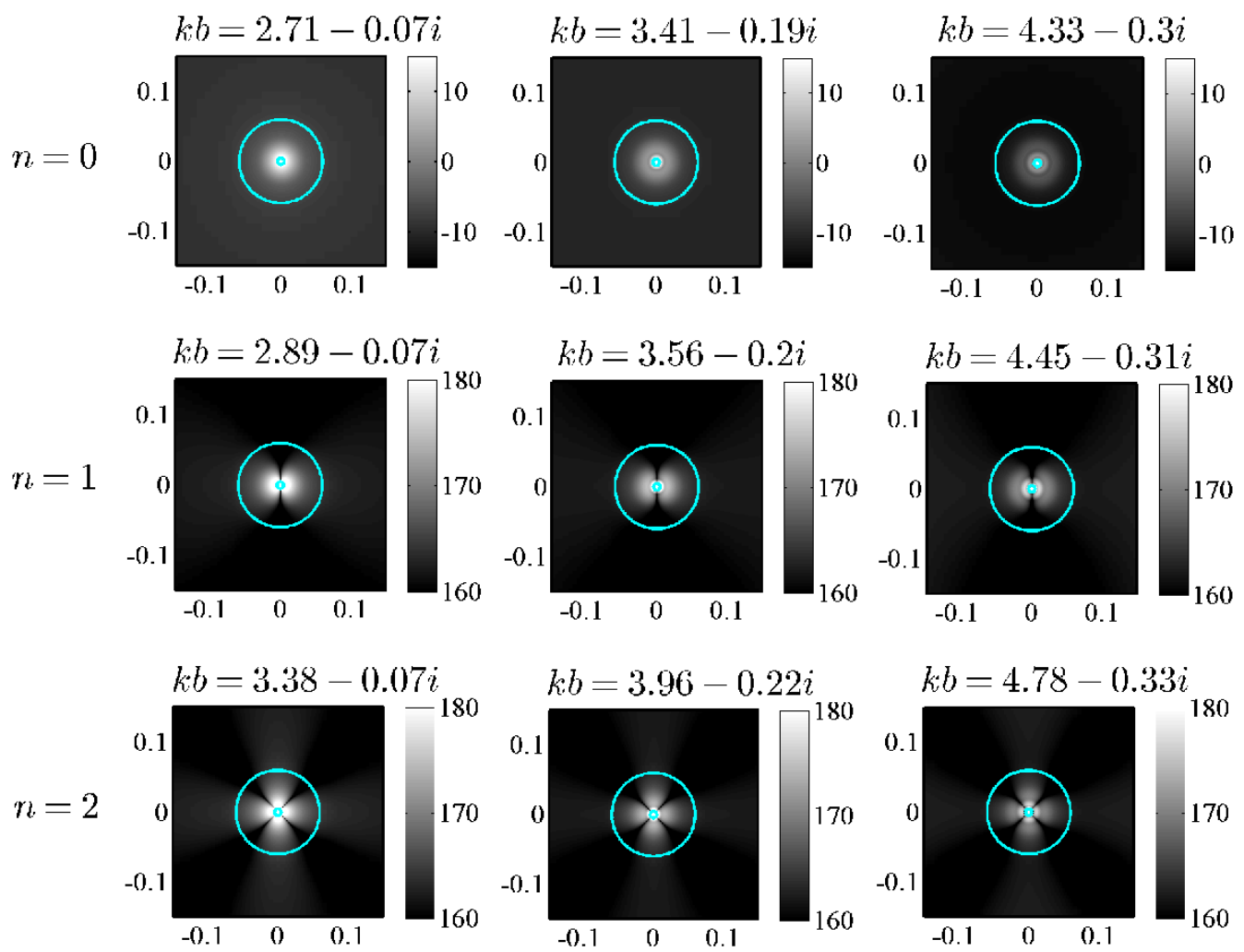

Figure 8: Modulus (dB scale) of the modal shape of the first trapped modes of an ABH. Three modes are shown for each circumferential order $n=0$ (first line), $n=1$ (second line) and $n=2$ (third line).

\subsection{Influence of the loss factor $\eta$}

In Fig. 9, the scattering cross-section of the ABH described on the table 1 is computed for various material loss factors. It is shown that the loss factor $\eta$ controls the amount of scattered energy, particularly at high frequencies for which the variations of $Q_{s c}$ are much smoother when $\eta$ increases : the value at the maxima can be divided by 2 when $\eta$ comes from $0.1 \%$ to $10 \%$. It is also shown that $\eta$ has a weak effect at low frequencies. As discussed in section 3.1, we define the transition between low and high frequencies by the condition $k_{c, 0} b \approx 2.42$ for the simulated $\mathrm{ABH}$, which leads to an incident wavelength $\lambda=\frac{2 \pi b}{2.42}$. For all values of $\eta$, the same first cut-on dimensionless wavenumber $k_{c, 0} b$ separates the low and high frequency ranges. From this 
obervations, a huge effect of the material loss factor can be expected while modelling an added damping layer.

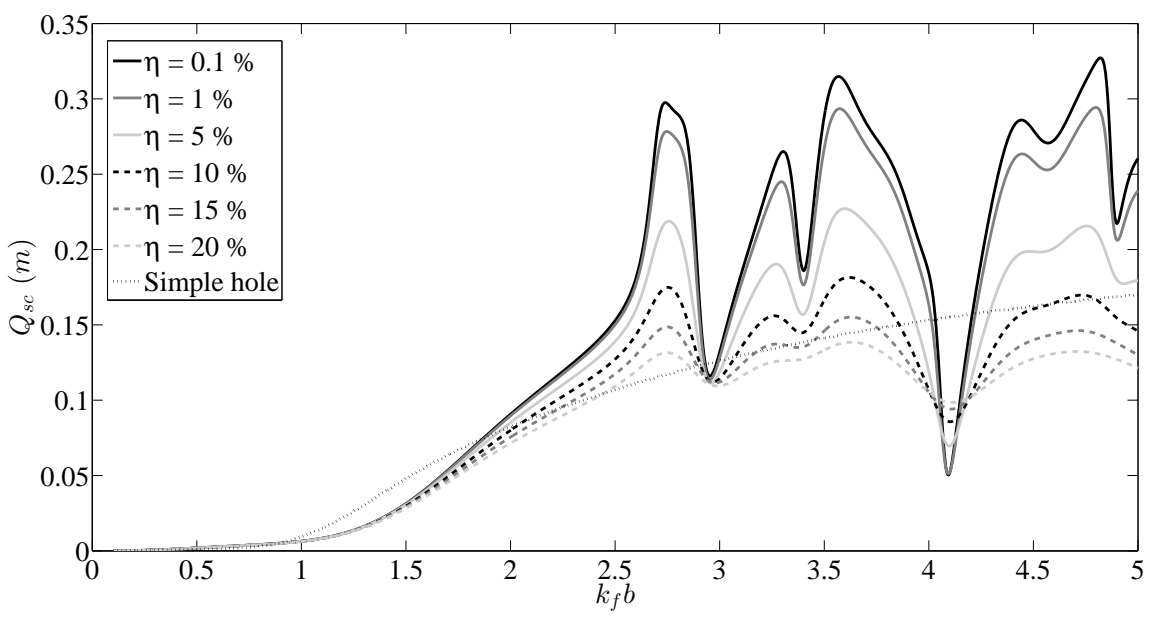

Figure 9: Influence of the loss factor $\eta$ on the scattering cross-section for a thickness ratio $h_{t} / h_{0}=1 / 100 . \quad h_{t}$ and $h_{0}$ represent the truncation thickness and the uniform thickness outside the $\mathrm{ABH}$.

\subsection{Influence of the truncation thickness $h_{t}$}

In the Fig. 10(b), scattering cross-section of the ABH described in table 1 is computed for various values of the thickness ratio $h_{t} / h_{0}$. Fig. 10(b) is plotted according to Eq. (31) by fixing the parameters $b, h_{0}$ and $a^{\prime}$ and varying the truncation thickness $h_{t}$ (see Fig. 10(a)). Quite expectingly, there is no significative effect of $h_{t}$ at low frequencies : as the cut-on frequency does not depend on $h_{t}$, the ABH's behave nearly the same wathever $h_{t}$. At high frequencies, $h_{t}$ being a driving parameter of the profile stiffness gradient, the location of the resonances versus frequency are largely modified. For example, the first resonance increases from $k_{f} b=2.73$ when $h_{t}=h_{0} / 100$ to $k_{f} b=3.84$ when $h_{t}=h_{0} / 10$. This short parametric study shows that the diameter of the central at a given profile (related to the quality of the ABH manufacturing) can play a major role in the scattered field outside the $\mathrm{ABH}$.

\subsection{Influence of the zero thickness radius $a^{\prime}$}

Fig. 11(b) shows the ABH scattering cross-section simulated for different values of the zero thickness radius $a^{\prime}$. This figure is plotted according to 


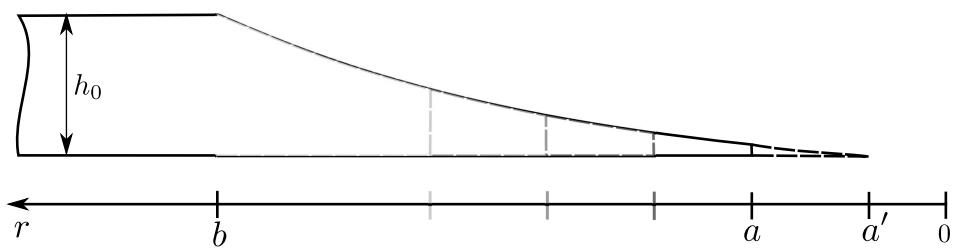

(a)

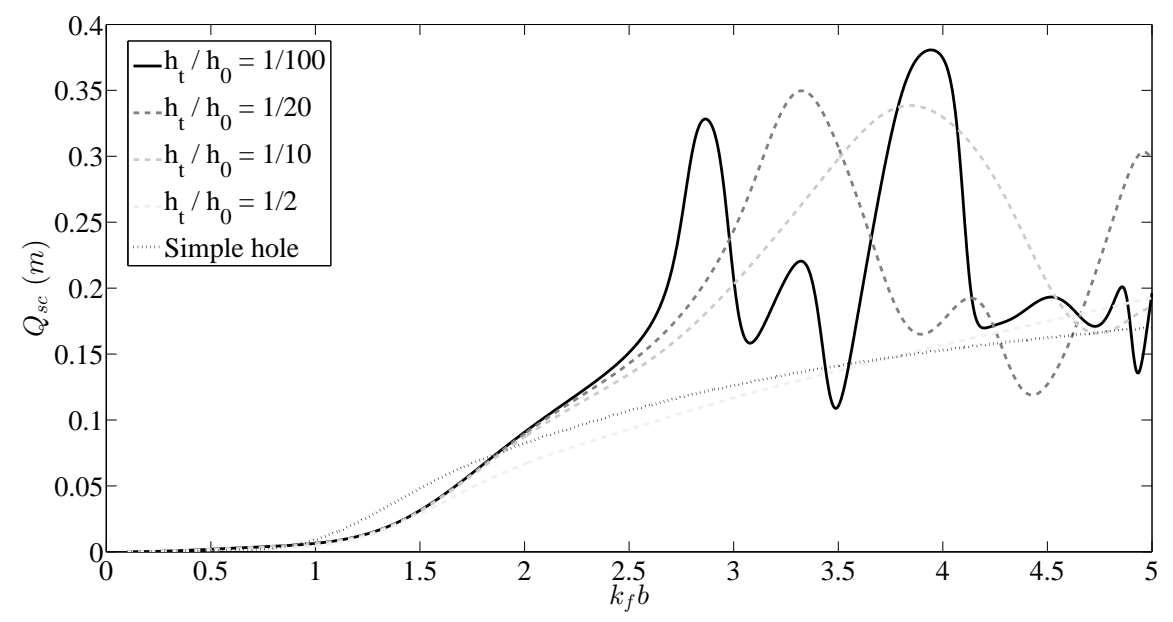

(b)

Figure 10: (a) Thickness profile of the $\mathrm{ABH}$ for different values of the thickness ratio $h_{t} / h_{0}$. (b) Effect of the truncation thickness $h_{t}$ on the scattering cross-section, with $b=6$ $\mathrm{cm}, h_{0}=1.5 \mathrm{~mm}$ and $a^{\prime}=1 \mathrm{~mm}$.

Eq.(31) by fixing the parameters $b, h_{0}$ and $h_{t}$ and varying the constant of the profile $a^{\prime}$, which varies the truncation radius $a$ (see Fig. 11(a)). As written in Eq. (24), $a^{\prime}$ is a driving parameter of the cut-on frequency such that it increases from $k_{c, 0} b=2.43$ for $a^{\prime}=1.10^{-3} \mathrm{~m}$ to $k_{c, 0} b=3.58$ for $a^{\prime}=2 \cdot 10^{-2} \mathrm{~m}$. As a result, all being equal, the resonances are just shifted to higher frequencies. This study shows that the control of the fastness or slowness of the stiffness gradient of the profile allows to tune the $\mathrm{ABH}$ resonances on selected frequencies. 


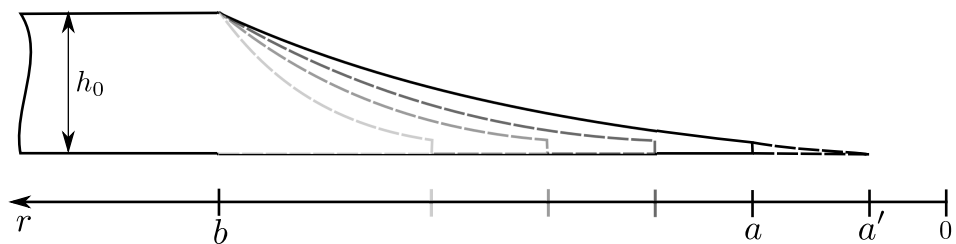

(a)

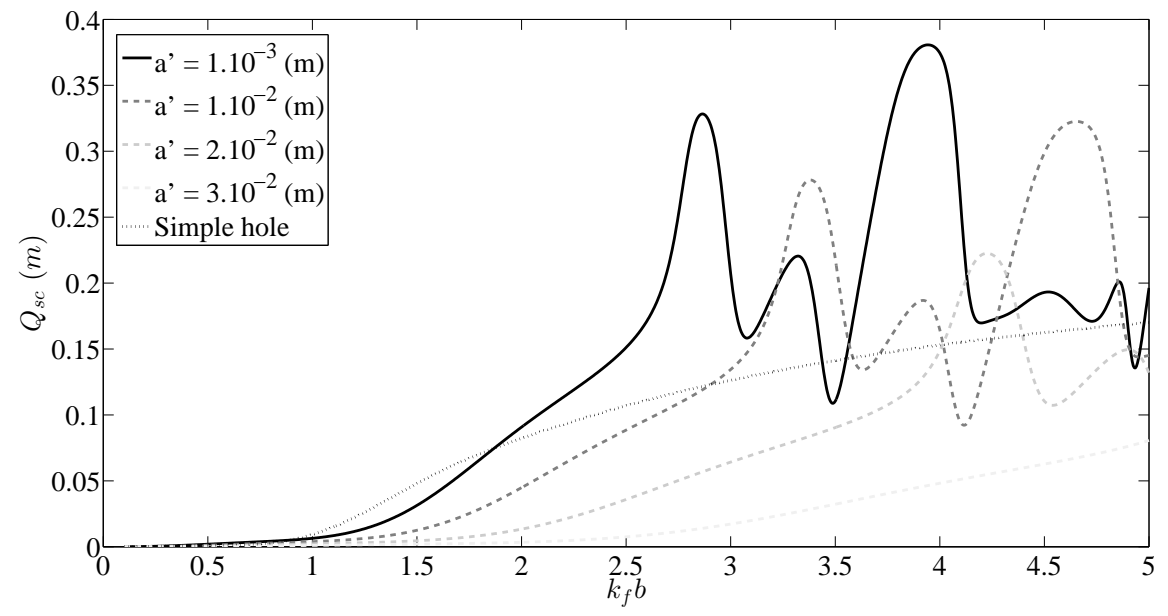

(b)

Figure 11: (a) Thickness profile of the ABH for different values of the zero thickness radius $a^{\prime}$. (b) Influence of $a^{\prime}$ on the scattering cross-section, with $b=6 \mathrm{~cm}, h_{0}=1.5 \mathrm{~mm}$ and $h_{t}=15 \mu \mathrm{m}$.

\section{Conclusion}

In this paper, the scattering of flexural waves from an acoustic black hole, seen as a penetrable scatterer, is modelled within the framework of the Kirchhoff theory. In the case of a quadratic profile $(m=2)$ with uniform internal damping properties, the analytical derivation of the wave dispersion relations inside the ABH is a major outcome of the model. Cut-on frequencies are analytically obtained, giving thus theoretical interpretation of the threshold frequency for damping vibration observed experimentally and numerically in previous works. It is shown that for each circumferential order, above the cut-on frequency, two waves become propagative inside the $\mathrm{ABH}$, leading to the apparition of $\mathrm{ABH}$ trapped modes. The analysis of the response of the 
$\mathrm{ABH}$ to an incident bending plane wave from the modal point of vue gives new insights to the understanding of $\mathrm{ABH}$. When simulating the scattered field and scattering cross-section, it is shown that below the first cut-on dimensionless wave number $\left(k_{c, 0} b\right)$, the $\mathrm{ABH}$ cross-section behaves as a simple hole. In the high frequency range (above $k_{c, 0} b$ ), the $\mathrm{ABH}$ exhibits maximal scattering effect at given frequencies related to resonances of its trapped modes. Parametric studies of three ABH material or geometrical parameters are also led. It is shown that the material loss factor $\eta$ tends to reduce the resonant behaviour of the scatterer, particularly in the high frequency range. Also, the truncation thickness $h_{t}$ and the zero thickness radius $a^{\prime}$, which set the stiffness gradient, can shift the $\mathrm{ABH}$ resonance frequencies. In order to be able to thinly control the scattering properties of the $\mathrm{ABH}$ and taylor it to mechanical engineering applications, further investigations would be led to take into account a covering damping layer, to model any thickness profile with or without central hole, and to extend the model to multiple scattering. 


\section{APPENDIX}

In appendices $\mathrm{A}$ and $\mathrm{B}$, useful details for computing the scattered fields of the simple hole and the acoustic black hole are given.

\section{Appendix A. Simple hole configuration}

The displacement field in a plate containing a simple hole is given as,

$$
W^{e x t}=W_{0} \sum_{n=0}^{\infty} \varepsilon_{n} i^{n} J_{n}\left(k_{f} r\right) \cos (n \theta)+\sum_{n=0}^{\infty}\left[A_{n} H_{n}^{(1)}\left(k_{f} r\right)+B_{n} K_{n}\left(k_{f} r\right)\right] \cos (n \theta), \quad r>b .
$$

Using Eq. (7) and Eq. (9), the bending moment and the transverse force in the plate are written as

$$
\begin{aligned}
M\left(W^{e x t}\right)= & -D_{0} \sum_{n=0}^{\infty}\left\{W_{0} \varepsilon_{n} i^{n}\left[\left(\frac{n^{2}(1-\nu)-\left(k_{f} r\right)^{2}}{r^{2}}\right) J_{n}\left(k_{f} r\right)-\left(\frac{k_{f} r(1-\nu)}{r^{2}}\right) J_{n}^{\prime}\left(k_{f} r\right)\right]\right. \\
& +A_{n}\left[\left(\frac{n^{2}(1-\nu)-\left(k_{f} r\right)^{2}}{r^{2}}\right) H_{n}^{(1)}\left(k_{f} r\right)-\left(\frac{k_{f} r(1-\nu)}{r^{2}}\right) H_{n}^{(1)^{\prime}}\left(k_{f} r\right)\right] \\
& \left.+B_{n}\left[\left(\frac{n^{2}(1-\nu)+\left(k_{f} r\right)^{2}}{r^{2}}\right) K_{n}\left(k_{f} r\right)-\left(\frac{k_{f} r(1-\nu)}{r^{2}}\right) K_{n}^{\prime}\left(k_{f} r\right)\right]\right\} \cos (n \theta),
\end{aligned}
$$

$$
\begin{aligned}
V_{0}\left(W^{e x t}\right)= & -D_{0} \sum_{n=0}^{\infty}\left\{W_{0} \varepsilon_{n} i^{n}\left[\left(\frac{n^{2}(1-\nu)}{r^{3}}\right) J_{n}\left(k_{f} r\right)-k_{f} r\left(\frac{n^{2}(1-\nu)+\left(k_{f} r\right)^{2}}{r^{3}}\right) J_{n}^{\prime}\left(k_{f} r\right)\right]\right. \\
& +A_{n}\left[\left(\frac{n^{2}(1-\nu)}{r^{3}}\right) H_{n}^{(1)}\left(k_{f} r\right)-k_{f} r\left(\frac{n^{2}(1-\nu)+\left(k_{f} r\right)^{2}}{r^{3}}\right) H_{n}^{(1)^{\prime}}\left(k_{f} r\right)\right] \\
& \left.+B_{n}\left[\left(\frac{n^{2}(1-\nu)}{r^{3}}\right) K_{n}\left(k_{f} r\right)-k_{f} r\left(\frac{n^{2}(1-\nu)-\left(k_{f} r\right)^{2}}{r^{3}}\right) K_{n}^{\prime}\left(k_{f} r\right)\right]\right\} \cos (n \theta) .
\end{aligned}
$$

By applying free conditions Eq. (11) we get

$$
\left[\begin{array}{cc}
S_{H_{n}}(\alpha) & S_{K_{n}}(\alpha) \\
T_{H_{n}}(\alpha) & T_{K_{n}}(\alpha)
\end{array}\right] \cdot\left\{\begin{array}{c}
A_{n} \\
B_{n}
\end{array}\right\}=-W_{0} \varepsilon_{n} i^{n}\left\{\begin{array}{c}
S_{J_{n}}(\alpha) \\
T_{J_{n}}(\alpha)
\end{array}\right\} .
$$


The solutions of this system are obtained analytically:

$$
\begin{aligned}
& A_{n}=-W_{0} \varepsilon_{n} i^{n}\left[\frac{S_{J_{n}}(\alpha) T_{K_{n}}(\alpha)-S_{K_{n}}(\alpha) T_{J_{n}}(\alpha)}{S_{H_{n}}(\alpha) T_{K_{n}}(\alpha)-S_{K_{n}}(\alpha) T_{H_{n}}(\alpha)}\right], \\
& B_{n}=-W_{0} \varepsilon_{n} i^{n}\left[\frac{S_{H_{n}}(\alpha) T_{J_{n}}(\alpha)-S_{J_{n}}(\alpha) T_{H_{n}}(\alpha)}{S_{H_{n}}(\alpha) T_{K_{n}}(\alpha)-S_{K_{n}}(\alpha) T_{H_{n}}(\alpha)}\right],
\end{aligned}
$$

where

$$
\begin{gathered}
S_{X_{n}}(\alpha)=\left[n^{2}(1-\nu)-\alpha^{2}\right] X_{n}(\alpha)-(1-\nu) \alpha X_{n}^{\prime}(\alpha), \quad \alpha=k_{f} b, \\
S_{K_{n}}(\alpha)=\left[n^{2}(1-\nu)+\alpha^{2}\right] K_{n}(\alpha)-(1-\nu) \alpha K_{n}^{\prime}(\alpha), \\
T_{X_{n}}(\alpha)=\left[n^{2}(1-\nu)\right] X_{n}(\alpha)-\left[\alpha\left(n^{2}(1-\nu)+\alpha^{2}\right)\right] X_{n}^{\prime}(\alpha), \\
T_{K_{n}}(\alpha)=\left[n^{2}(1-\nu)\right] K_{n}(\alpha)-\left[\alpha\left(n^{2}(1-\nu)-\alpha^{2}\right)\right] K_{n}^{\prime}(\alpha) .
\end{gathered}
$$

The function $X_{n}$ represents the Bessel function of the first order $J_{n}$ or the Hankel function of the first kind $H_{n}^{(1)}$. The function $K_{n}$ is the second kind modified Bessel function of order $n$.

\section{Appendix B. Acoustic black hole configuration}

Displacement field, slope, bending moment and shear force calculated inside the $\mathrm{ABH}$ are given by:

$$
\begin{array}{r}
W^{\text {int }}=\sum_{n=0}^{\infty}\left\{\sum_{p=1}^{4}\left[C_{p, n}\left(\frac{\widehat{r}}{r_{0}}\right)^{i k_{p, n} r_{0}}\right]\right\} \cos (n \theta), \quad a<r<b, \\
\frac{\partial W^{i n t}}{\partial \widehat{r}}=\sum_{n=0}^{\infty}\left\{\sum_{p=1}^{4}\left[\frac{C_{p, n}}{\widehat{r}} i k_{p, n} r_{0}\left(\frac{\widehat{r}}{r_{0}}\right)^{i k_{p, n} r_{0}}\right]\right\} \cos (n \theta), \\
M\left(W^{i n t}\right)=-D^{i n t} \sum_{n=0}^{\infty}\left\{\sum_{p=1}^{4}\left[\frac{C_{p, n}}{\widehat{r}^{2}} E_{p, n}\left(\frac{\widehat{r}}{r_{0}}\right)^{i k_{p, n} r_{0}}\right]\right\} \cos (n \theta),
\end{array}
$$




$$
V\left(W^{i n t}\right)=-D^{i n t} \sum_{n=0}^{\infty}\left\{\sum_{p=1}^{4}\left[\frac{C_{p, n}}{\widehat{r}^{3}} F_{p, n}\left(\frac{\widehat{r}}{r_{0}}\right)^{i k_{p, n} r_{0}}\right]\right\} \cos (n \theta) .
$$

Applying continuity conditions in Eq. (5) and free conditions in Eq. (10), we get the following system:

$$
M . X=S
$$

where

$$
\begin{gathered}
\boldsymbol{M}=\left[\begin{array}{cccccc}
H_{n}^{(1)}(\beta) & K_{n}(\beta) & -1 & -1 & -1 & -1 \\
k_{f} H_{n}^{(1)^{\prime}}(\beta) & k_{f} K_{n}^{\prime}(\beta) & -i k_{1, n} & -i k_{2, n} & -i k_{3, n} & -i k_{4, n} \\
S_{H_{n}}(\beta) & S_{K_{n}}(\beta) & -E_{1, n} & -E_{2, n} & -E_{3, n} & -E_{4, n} \\
T_{H_{n}}(\beta) & T_{K_{n}}(\beta) & -F_{1, n} & -F_{2, n} & -F_{3, n} & -F_{4, n} \\
0 & 0 & G_{1, n} & G_{2, n} & G_{3, n} & G_{4, n} \\
0 & 0 & L_{1, n} & L_{2, n} & L_{3, n} & L_{4, n}
\end{array}\right] \\
\boldsymbol{X}=\left\{\begin{array}{c}
A_{n} \\
B_{n} \\
C_{1, n} \\
C_{2, n} \\
C_{3, n} \\
C_{4, n}
\end{array}\right\}, \quad \boldsymbol{S}=-W_{0} \varepsilon_{n} i^{n}\left\{\begin{array}{c}
J_{n}(\beta) \\
k_{f} J_{n}^{\prime}(\beta) \\
S_{J_{n}}(\beta) \\
T_{J_{n}}(\beta) \\
0 \\
0
\end{array}\right\}
\end{gathered}
$$

with

$$
\begin{gathered}
\beta=k_{f} r_{0} \text { with } r_{0}=b-a^{\prime}, \\
E_{p, n}=-k_{p, n}^{2} r_{0}^{2}+i k_{p, n} r_{0}(\nu-1)-\nu n^{2}, \\
F_{p, n}=-i k_{p, n}^{3} r_{0}^{3}-4 k_{p, n}^{2} r_{0}^{2}+\left[6(\nu-1)-n^{2}(2-\nu)\right] i k_{p, n} r_{0}+n^{2}(3-7 \nu), \\
G_{p, n}=E_{p, n}\left[\frac{t}{r_{0}}\right]^{i k_{p, n} r_{0}}, \quad L_{p, n}=F_{p, n}\left[\frac{t}{r_{0}}\right]^{i k_{p, n} r_{0}} \text { with } t=a-a^{\prime} .
\end{gathered}
$$

Solving Eq. (B.5) provide the unknown $\boldsymbol{X}$. 


\section{References}

[1] M. A. Mironov, Propagation of a flexural wave in a plate whose thickness decreases smoothly to zero in a finite interval, Soviet Physics 34 (3) (1988) 318-319.

[2] V. Krylov, F. Tilman, Acoustic 'black holes' for flexural waves as effective vibration dampers, Journal of Sound and Vibration 274 (3) (2004) 605-619.

[3] V. V. Krylov, R. Winward, Experimental investigation of the acoustic black hole effect for flexural waves in tapered plates, Journal of Sound and Vibration 300 (1) (2007) 43-49.

[4] D. O'Boy, V. V. Krylov, V. Kralovic, Damping of flexural vibrations in rectangular plates using the acoustic black hole effect, Journal of Sound and Vibration 329 (22) (2010) 4672-4688.

[5] F. Gautier, J. Cuenca, V. V. Krylov, L. Simon, Experimental investigation of the acoustic black hole effect for vibration damping in elliptical plates, Acoustics'08, Paris June 30 - July 4, 2008.

[6] J. Cuenca, Wave models for the flexural vibrations of thin plates, Ph.D. thesis, Université du Maine, Le Mans, France (2009).

[7] V. Georgiev, J. Cuenca, F. Gautier, L. Simon, V. Krylov, Damping of structural vibrations in beams and elliptical plates using the acoustic black hole effect, Journal of sound and vibration 330 (11) (2011) 24972508.

[8] P. Morse, H. Feshbach, Methods of theoretical physics, Vol. 1,2, McGraw-Hill, 1953.

[9] C. Mow, Y. Pao, The diffraction of elastic waves and dynamic stress concentrations, Taylor and Francis Group, 1973.

[10] A. Norris, C. Vemula, Scattering of flexural waves on thin plates, Journal of sound and vibration 181 (1) (1995) 115-125.

[11] C. Vemula, A. Norris, Flexural wave propagation and scattering on thin plates using mindlin theory, Wave Motion 26 (1) (1997) 1-12. 
[12] V. A. Squire, T. W. Dixon, Scattering of flexural waves from a coated cylindrical anomaly in a thin plate, Journal of Sound and Vibration 236 (2) (2000) 367-373.

[13] D. O'Boy, V. Krylov, Damping of flexural vibrations in circular plates with tapered central holes, Journal of Sound and Vibration 330 (10) (2011) 2220-2236.

[14] F. Fahy, P. Gardonio, Sound and Structural Vibration, 2nd Edition, Elsevier, 2007.

[15] K. Liew, C. Wang, Y. Xiang, S. Kitipornchai, Vibration of Midlin plates, Elsevier Science Ltd, 1998.

[16] H.-N. Chu, G. Hermann, Influence of large amplitudes on free flexural vibrations of rectangular elastic plates, Journal of applied mechanics 23 (1956) 532-540.

[17] A. Leissa, Vibration of Plates, N70-18461, NASA, 1969.

[18] E. T. Whittaker, G. N. Watson, A course of modern analysis: an introduction to the general theory of infinite processes and of analytic functions, with an acount of the principal transcendental functions, University Press, 1935.

[19] M. Abramowitz, I. Stegun, Handbook of mathematical functions: with formulas, graphs, and mathematical tables, Vol. 55, Dover publications, 1965 .

[20] H. Conway, Some special solutions for the flexural vibration of discs of varying thickness, Archive of Applied Mechanics 26 (6) (1958) 408-410.

[21] E. Durand, Solutions numériques des équations algébriques [Numerical solutions of algebraic equations], Paris: Masson, 1971.

[22] V. Denis, A. Pelat, F. Gautier, B. Elie, Modal overlap factor of a beam with an acoustic black hole termination, Journal of Sound and Vibration 333 (12) (2014) 2475-2488.

[23] M. Callan, C. Linton, D. Evans, Trapped modes in two-dimensional waveguides, Journal of Fluid Mechanics 229 (1991) 51-64. 
[24] P. Cobelli, V. Pagneux, A. Maurel, P. Petitjeans, Experimental observation of trapped modes in a water wave channel, EPL (Europhysics Letters) 88 (2) (2009) 20006.

[25] R. Porter, Trapped waves in thin elastic plates, Wave Motion 45 (1) (2007) 3-15.

[26] O. Xeridat, Etude expérimentale de la propagation, de la diffusion et de la localisation des ondes de lamb [experimental study of the propagation, the scattering and the localization of lamb waves], Ph.D. thesis, Université de Nice Sophia-Antipolis (2011). 